\title{
Technology to Assess Sleep
}

Editor

THOMAS PENZEL

\section{SLEEP MEDICINE CLINICS}

www.sleep.theclinics.com

Consulting Editor

TEOFILO LEE-CHIONG Jr

December 2016 • Volume 11 • Number 4 